\title{
Anterolateral Approach Might Be Superior to Posterolateral Approach in Controlling Lower Limb Discrepancy for Special Total Hip Arthroplasty Patients
}

\section{Ao Xiong}

Peking University Shenzhen Hospital

Guoqing Li

Peking University Shenzhen Hospital

\section{Su Liu}

Peking University Shenzhen Hospital

\section{Chang Xu}

Peking University Shenzhen Hospital

\section{Deli Wang}

Peking University Shenzhen Hospital

Hui Zeng ( $\square$ zenghui_36@163.com )

Peaking University Shenzhen Hospital https://orcid.org/0000-0001-6966-0769

\section{Research Article}

Keywords: anterolateral approach (ALA), posterolateral approach (PLA), total hip arthroplasty (THA), lower limb discrepancy (LLD), radiological

Posted Date: August 9th, 2021

DOI: https://doi.org/10.21203/rs.3.rs-769196/v1

License: (c) (1) This work is licensed under a Creative Commons Attribution 4.0 International License. Read Full License 


\section{Abstract}

Objectives: Variable approaches for total hip arthroplasty (THA) are promoted but investigations of associations between the approaches and the postoperative lower limb discrepancy (LLD) are limited. We intended to compare the efficacy between the anterolateral approach (ALA) and posterolateral approach $(\mathrm{PLA})$ in controlling of postoperative LLD.

Methods: Two retrospective cohorts were established including patients undergone unilateral primary THA. Preoperative and latest follow-up radiographs were compared and evaluated. Two hundred and sixty patients ( 140 females and 126 males; aged $46.70 \pm 13.62$ years) were recruited with around 20 months follow-up postoperatively.

Results: Univariate analysis identified lower postoperative LLD, lower postoperative acetabular offset and lower hospital costs in the ALA group than in the PLA group ( $\mathrm{\otimes} \otimes 0.01)$, however, the femoral and global offset and length of stay (LOS) are comparable between $(p>0.05)$. Multivariable liner regression revealed that PLA, preoperative LLD, developmental dysplasia of the hip and aseptic avascular necrosis are major contributors to the postoperative LLD $(p<0.05)$.

Conclusions: This study illustrates that the ALA might be superior to the PLA in controlling the postoperative LLD among some of the THA patients. Both ALA and PLA are comparable in regard to femoral and offset and LOS.

\section{Introduction}

Total hip arthroplasty (THA) is a valid method for various hip diseases such hip osteoarthritis (HOA), aseptic avascular necrosis (AVN) of the femoral head, developmental dysplasia of the hip (DDH), and femoral neck fracture (FNF) [1, 2]. The clinical efficacy of THA has been significantly improved with the developments of relevant technologies with the 15-year survival rate of the prosthesis over $95 \%$ [3]. Large quantity of THAs are necessary in the setting of the aging population [4]. Successful THA can provide adequate pain relief, restore hip function, improve quality of life, and obtain satisfactory long-term outcomes [5].

Postoperative lower limb discrepancy (LLD) and improper offset occur occasionally, which might result in the adverse events, instability, impingement, or even dislocation of the prosthesis [6, 7]. The LLD also remains the most common cause of patient dissatisfaction and postoperative gait disorders [8]. The improper offset also unbalances the arm of the abduction muscle to weaken the strength, increases the pressure of the hip joint, and accelerates the wear of prosthesis [9].

Anterolateral approach (ALA) and posterolateral approach (PLA) are the two commonly applied THA approaches $[10,11]$. The ALA is performed thought the gluteus medius to increase the hip load and abduction with reproduceable results and to decrease the dislocation rates $[12,13]$. The PLA is an approach with an excellent exposure view by damaging joint capsule with shorter duration, lower 
intraoperative fracture but higher risk of dislocation, compared with direct anterior approaches [14, 15]. To the best of our knowledge, the efficacy of either ALA or PLA to control the postoperative LLD and to restore the offset has not been sufficiently investigated. Therefore, the present study aimed to investigate the possible association of surgical approaches (ALA or PLA) with postoperative LLD and offset in primary unilateral THAs.

\section{Materials And Methods}

\section{Participations}

The present study was carried out in Peking University Shenzhen Hospital located in South China between October 2018 and July 2019. The study protocol was approved by the Ethics Committee of Peking University Shenzhen Hospital (No. 2019038) and all patients gave their inform and consent. Patients undergone the unilateral primary THA via the ALA or the PLA were retrospectively included. Since radiographic evaluation is probably unreliable for FNF patients, only patients who were diagnosed as HOA, DDH, inflammatory arthritis, and AVN were recruited. Patients who failed to understand or signed consent, lost follow-up or missed information, or obtained unqualified hip radiographs were excluded. The exclusion criteria were illustrated in the flowchart (Figure. 1). All participations were invited for postoperative clinical and radiological follow-ups. All operations were conducted by the experienced and skilled surgeons specialized in arthroplasty with over 10-year experience of both the ALA and PLA approaches (over 200 arthroplasty operations annually).

\section{Variables}

The discharge notes of all patients were reviewed and relevant variables were recorded including their basic demographic information and radiographic parameters. Basic demographic information included the gender, age, height, weight, body mass index (BMI), marriage, hospital cost, length of stay (LOS), diagnosis, and American Society of Anesthesiologists (ASA) score [4]. Radiographic outcomes included the postoperative LLD, femoral offset (FO), acetabular offset (AO), and global offset (GO) [11]. LOS was defined as the periods between the admitted date to discharge date [16]. The extraction differences, if existing, were resolved by discussing with a third party and the data were independently extracted by two investigators (GQL and SL).

\section{Radiological assessment}

The preoperative and postoperative plain radiographs were performed in the anteroposterior and lateral positions for both hip joints routinely for THA patients and archived in the Picture Archiving and Communication System (PACS) (Tomorrow Medical Network Science and Technology, Ningbo, Zhejiang, China). These historical radiographs retrieved from PACS were evaluated by the senior surgeons (AX and DLW) to confirm the qualification elsewise a new one would be filmed. The LLD was measured between both the teardrop lines and the lesser trochanter base and the absolute value represented the distance of the THA side compared to the untreated side as described elsewhere [17]. The FO was defined as the 
distance between the center of rotation (COR) and the femoral axis. The AO was defined as the distance between the COR and the tear drop axis (Figure. 2). The GO was measured as the distance between the femur longitudinal axis to the COR plus the distance from the COR to a vertical line of the medial edge of the ipsilateral teardrop point of the pelvis [2]. To reduce the bias of measurements, all radiographic variables were measured and recorded as continuous variables by two independent observers (GQL and $\mathrm{SL}$ ) with a standardized process. Inter-observer and intra-observer reliabilities of the afore-mentioned radiological measurements were evaluated.

\section{Statistical analysis}

All statistical analysis was performed by the R software 4.0.2 (R Foundation for Statistical Computing, Vienna, Austria) [18]. Continuous variables were summarized by mean \pm standard deviation (SD) while categorical variables were summarized by number and percentage. For the continuous variables and data with a normal distribution, Student-t test was applied. For the categorical variables, Chi-square test and Fisher's exact test were applied properly according to the expected values greater than five or not. Univariate analysis was used to analyze the relationship of clinical outcomes and variables. Multivariate linear regression analysis was performed to examine the significant factors on postoperative LLD. All statistics were 2-tailed. With $p<0.05$ as the statistical significance [19] and reported with $95 \%$ confidence interval (Cl).

\section{Results}

According to the inclusion and exclusion criteria, 266 patients (140 females and 126 males) were included for the in-depth analyses and categorized into the ALA group $(\mathrm{N}=66)$ and the PLA group ( $N=$ 200). All enrolled participations were aged $46.70 \pm 13.60$ (range, 22.00 to 90.00 ) years old with mean BMI $22.73 \pm 3.28$ (range, 14.53 to 35.16 ) $\mathrm{kg} / \mathrm{m}^{2}$ and followed up for an average of 20 months. Three cases with dislocation in PLA group were cured with the conservative treatments, while no dislocation case occurred in the ALA group. No other postoperative complications (e.g. periprosthetic fracture and infection) and readmission within the follow-up period were reported within either group. All patients reported satisfactory hip function and normal muscle strength at the last follow-up.

The demographic characteristics were generally comparable between both groups including baseline variables and preoperative radiographs parameters (Pre-op LLD ) ( $p \otimes 0.05)$ apart from gender $(p \otimes 0.01)$ and age $(p \otimes 0.01)$ (Table 1). Preoperative LLD was measured as $13.40 \pm 17.50 \mathrm{~mm}$ and $12.17 \pm 14.89 \mathrm{~mm}(p=$ 0.609 ) in the ALA group and the PLA group, respectively. Patients with the DDH diagnosis (48 females and 33 males) were recorded to be 59 and 22 in the PLA and ALA groups respectively. 
Table 1

Demographic of participations between the two groups $(\mathrm{N}=266)$

\begin{tabular}{|c|c|c|c|c|}
\hline Variables & Categories & $\operatorname{ALA}(N=66)$ & $\operatorname{PLA}(N=200)$ & $P$ Value \\
\hline \multirow[t]{2}{*}{ Gender ${ }^{a}$} & Female & $43(65.15 \%)$ & $97(48.50 \%)$ & \multirow[t]{2}{*}{$0.03^{c *}$} \\
\hline & Male & $23(34.85 \%)$ & $103(51.50 \%)$ & \\
\hline Age $^{b}$ & Mean \pm SD & $53.87 \pm 13.96$ & $44.33 \pm 12.68$ & $\llbracket 0.01^{\star \star}$ \\
\hline Height ${ }^{b}$ & Mean \pm SD & $1.62 \pm 0.09$ & $1.64 \pm 0.08$ & 0.089 \\
\hline Weight ${ }^{b}$ & Mean \pm SD & $59.64+10.48$ & $61.10+11.47$ & 0.337 \\
\hline $\mathrm{BMI} \mathrm{b}^{\mathrm{b}}$ & Mean \pm SD & $22.73 \pm 2.73$ & $22.73 \pm 3.45$ & 0.986 \\
\hline \multirow[t]{2}{*}{ Marriage $^{a}$} & Single & $18(27.27 \%)$ & $48(24.00 \%)$ & \multirow[t]{2}{*}{$0.712^{c}$} \\
\hline & Couple & $48(72.73 \%)$ & $152(76.00 \%)$ & \\
\hline \multirow{4}{*}{ Diagnosis $^{a}$} & $\mathrm{HOA}$ & $10(15.15 \%)$ & $39(19.50 \%)$ & \multirow[t]{4}{*}{$0.727^{d}$} \\
\hline & $\mathrm{DDH}$ & $22(33.33 \%)$ & $59(29.50 \%)$ & \\
\hline & Inflammatory & $3(4.55 \%)$ & $6(3.00 \%)$ & \\
\hline & AVN & $31(46.97 \%)$ & $96(48.00 \%)$ & \\
\hline \multirow[t]{3}{*}{$\mathrm{ASA}^{\mathrm{a}}$} & 1 & $29(43.94 \%)$ & $108(54.00 \%)$ & \multirow[t]{3}{*}{$0.329^{d}$} \\
\hline & 2 & $35(53.03 \%)$ & $87(43.50 \%)$ & \\
\hline & 3 & $2(3.03 \%)$ & $5(2.50 \%)$ & \\
\hline Pre-op LLD b & Mean \pm SD & $13.40 \pm 17.50$ & $12.17 \pm 14.89$ & 0.609 \\
\hline \multirow{2}{*}{\multicolumn{5}{|c|}{$\begin{array}{l}\text { Abbreviations: ALA, anterior lateral approach; ASA, American Society of Anesthesiologists; AVN, } \\
\text { aseptic vascular necrosis; BMI, body mass index; CHD, coronary heart disease; DDH, developmental } \\
\text { dysplasia of the hip; HOA, hip osteoarthritis; LLD, leg length discrepancy; N, number; pre-op, } \\
\text { preoperative; PLA, posterior lateral approach; SD, standard deviation; THA, total hip arthroplasty. }\end{array}$}} \\
\hline \multicolumn{4}{|c|}{$\star, p<0.05, * *, p<0.01$} & \\
\hline \multicolumn{5}{|c|}{ a The values of categorical statistics are given as the number and percentage (\%) of patients. } \\
\hline \multicolumn{5}{|c|}{ b The values of continuous statistics are given as the mean and the standard deviation. } \\
\hline \multicolumn{5}{|c|}{${ }^{\mathrm{c}}$ Pearson's Chi-squared test. } \\
\hline${ }^{d}$ Fisher's Exa & & & & \\
\hline
\end{tabular}

The univariate analysis identified, compared with the PLA group, significant decreases of postoperative LLD ( $\$ \varangle 0.01 ; 95 \% \mathrm{Cl},-6.73 \mathrm{~mm}$ to $-2.49 \mathrm{~mm}$ ), postoperative acetabular offset ( $p \varangle 0.01 ; 95 \% \mathrm{Cl},-4.78 \mathrm{~mm}$ to 
$-1.43 \mathrm{~mm})$, and hospital costs ( $p \otimes 0.01 ; 95 \% \mathrm{Cl},-18882.90 \mathrm{RMB}$ to $-5731.24 \mathrm{RMB})$ in the ALA group. However, the LOS $(p=0.080)$, postoperative FO $(p=0.084)$, and postoperative GO $(p=0.067)$ are comparable between both groups (Table 2 ). 
Table 2

Comparison of postoperative outcomes between the two groups $(\mathrm{N}=266)$

\begin{tabular}{|c|c|c|c|c|c|c|}
\hline Parameters & Unit & $\operatorname{ALA}(\mathrm{N}=66)$ & $\operatorname{PLA}(\mathrm{N}=200)$ & $t$ value & $95 \% \mathrm{Cl}$ & $\begin{array}{l}P \\
\text { Value }\end{array}$ \\
\hline Costs $^{a}$ & Yuan & $\begin{array}{l}89060.83 \pm \\
19253.30\end{array}$ & $\begin{array}{l}101367.90 \pm \\
33166.13\end{array}$ & -3.69 & $\begin{array}{l}(-18882.90 \\
-5731.24)\end{array}$ & $\otimes 0.01^{* *}$ \\
\hline $\operatorname{LOS}^{a}$ & day & $15.59 \pm 8.31$ & $17.56 \pm 6.19$ & -1.77 & $(-4.17,0.24)$ & 0.080 \\
\hline $\begin{array}{l}\text { Post-op LLD } \\
\text { a }\end{array}$ & $\mathrm{mm}$ & $6.43 \pm 8.19$ & $11.04 \pm 11.71$ & -4.29 & $(-6.73,-2.49)$ & $\otimes 0.01 * *$ \\
\hline Post-op FO a & $\mathrm{mm}$ & $8.13 \pm 6.88$ & $10.02 \pm 9.57$ & -1.73 & $(-4.03,0.26)$ & 0.084 \\
\hline Post-op AO a & $\mathrm{mm}$ & $6.01 \pm 5.19$ & $9.12 \pm 7.91$ & -3.66 & $(-4.78,-1.43)$ & $\nabla 0.01 * \star$ \\
\hline Post-op GO a & $\mathrm{mm}$ & $11.22 \pm 8.24$ & $13.84 \pm 14.01$ & -1.84 & $(-5.41,0.18)$ & 0.067 \\
\hline \multicolumn{7}{|c|}{$\begin{array}{l}\text { Abbreviations: ALA, anterolateral approach; } \mathrm{AO} \text {, acetabular offset; } \mathrm{Cl} \text {, confidence interval; } \mathrm{FO} \text {, femoral } \\
\text { offset; GO, global offset; LLD, leg length discrepancy; } \mathrm{N} \text {, number; post-op, postoperative; PLA, posterior } \\
\text { lateral approach; S.E, standard error; THA, total hip arthroplasty. }\end{array}$} \\
\hline \multicolumn{7}{|l|}{${ }^{* *} p<0.01}$. \\
\hline \multicolumn{7}{|c|}{ a The values of continuous statistics are given as the mean and the standard deviation. } \\
\hline \multicolumn{7}{|c|}{$\begin{array}{l}\text { Table } 3 \text { Multivariable liner regression analysis of postoperative LLD ( } N \\
=266)\end{array}$} \\
\hline Variables & $\beta$ & $S E$ & $t$ Value & $95 \% \mathrm{Cl}$ & $P$ Value & \\
\hline Age & -0.02 & 0.05 & -0.39 & $\begin{array}{l}(-0.11 \\
0.08)\end{array}$ & 0.694 & \\
\hline \multicolumn{7}{|l|}{ Gender } \\
\hline Female & \multicolumn{5}{|c|}{ (Reference) } & \\
\hline Male & -1.38 & 1.25 & -1.10 & $\begin{array}{l}(-3.84 \\
1.08)\end{array}$ & 0.271 & \\
\hline BMI & 0.30 & 0.19 & 1.54 & $\begin{array}{l}(-0.08 \\
0.67)\end{array}$ & 0.126 & \\
\hline \multicolumn{7}{|l|}{ Diagnosis } \\
\hline $\mathrm{HOA}$ & \multicolumn{5}{|c|}{ (Reference) } & \\
\hline DDH & 5.01 & 1.79 & 2.79 & $\begin{array}{l}(1.47 \\
8.55)\end{array}$ & $0.005^{\star *}$ & \\
\hline Inflammation & 3.37 & 3.63 & 0.93 & $\begin{array}{l}(-3.78 \\
10.53)\end{array}$ & 0.354 & \\
\hline
\end{tabular}




\begin{tabular}{|c|c|c|c|c|c|}
\hline Parameters & Unit & $\operatorname{ALA}(N=66)$ & $\operatorname{PLA}(N=200)$ & $t$ value & $95 \% \mathrm{Cl}$ \\
\hline AVN & 3.81 & 1.68 & 2.27 & $\begin{array}{l}(0.50 \\
7.12)\end{array}$ & $0.024 *$ \\
\hline \multicolumn{6}{|l|}{ Approach } \\
\hline ALA & \multicolumn{5}{|c|}{ (Reference) } \\
\hline PLA & 4.71 & 1.48 & 3.17 & $\begin{array}{l}(1.78 \\
7.64)\end{array}$ & $0.001 * *$ \\
\hline Pre-op LLD & 0.29 & 0.04 & 7.34 & $\begin{array}{l}(0.21 \\
0.37)\end{array}$ & $<0.01^{\star \star}$ \\
\hline \multicolumn{6}{|c|}{$\begin{array}{l}\text { Abbreviations: ALA, anterolateral approach; AVN, avascular necrosis; BMI, body mass } \\
\text { index; Cl, confidence interval; DDH, developmental dysplasia of the hip; HOA, hip } \\
\text { osteoarthritis; LLD, leg length discrepancy; N, number; PLA, posterior lateral approach; pre- } \\
\text { op, preoperative; S.E, standard error. }\end{array}$} \\
\hline \multicolumn{6}{|c|}{${ }^{\star} p<0.05 ; * \star p<0.01}$. \\
\hline \multicolumn{6}{|c|}{ Adjust variables: age, gender, diagnosis, approach, and preoperative LLD. } \\
\hline
\end{tabular}

The multivariate liner regression revealed that the approach type (PLA, $p=0.001 ; \beta=4.71 ; 95 \% \mathrm{Cl}, 1.78$ to 7.64), preoperative LLD ( $p<0.01 ; \beta=0.29 ; 95 \% \mathrm{Cl}, 0.21$ to 0.37 ), diagnosis ( $\mathrm{DDH}, p=0.005 ; \beta=5.01 ; 95 \%$ $\mathrm{Cl}, 1.47$ to 8.55 ) and (AVN, $p=0.044 ; \beta=3.81 ; 95 \% \mathrm{Cl}, 0.50$ to 7.12 ) were substantial contributors to the postoperative LLD. However, no significant differences were observed among age $(p=0.694 ; \beta=-0.02$; $95 \% \mathrm{Cl},-0.11 \mathrm{~mm}$ to $0.08 \mathrm{~mm}$ ), gender (male; $\beta=-1.38,95 \% \mathrm{Cl},-3.84 \mathrm{~mm}$ to $1.08 \mathrm{~mm}$ ), and $\mathrm{BMI}(\beta=0.30$, $95 \% \mathrm{Cl},-0.08 \mathrm{~mm}$ to $0.67 \mathrm{~mm}$ ) (Table 3 ).

Regression curves of postoperative discrepancy and preoperative LLD between the two groups displayed in Figure. 3a. Obviously lower postoperative discrepancy in the ALA group than that in the PLA group ( $p<$ $0.01)$. The regression curves of postoperative discrepancy and diagnosis and BMI were displayed in Figure. $3 \mathrm{~b}$ and Figure. 3c.

Regression curves of preoperative offset and postoperative offset (AO (a), FO (b), and GO (c)) (Figure. 4), which implied that they were comparable between the two groups.

\section{Discussion}

The present study showed that the ALA might be superior to the PLA in controlling the short-term postoperative LLD among some of the unilateral primary THA patients. However, their postoperative FO, GO restoration, and LOS are comparable. Both approaches achieved satisfactory joint function within the 20-month follow-up. 
Our most import finding is that the ALA might be superior to the PLA in controlling postoperative LLD. The univariate analyses revealed the postoperative LLD were significantly lower and more stable in the ALA group than in the PLA group over 20 months postoperatively. Similarly, multivariate liner regression analysis also revealed that THA approach is significantly associated with postoperative LLD. Notable relationships between discrepancy and approaches were reflected with a higher discrepancy in the PLA group ( $p=0.001 ; \beta=4.71 ; 95 \% \mathrm{Cl}, 1.78$ to 7.64$)$. These results are highly consistent with several earlier studies [20]. A previous study demonstrated that the accurate reconstruction of biomechanical parameters related to the limb discrepancy after THAs is critical to ensure a long-term success [21]. Nevertheless, discrepancies after THAs may necessitate subsequent interventions but current methods during the operation are either inaccurate or expensive [22]. Intelligent HIP smart tool, a newly introduced smart tool providing an accurate and real-time intraoperative leg length measurements, is properly useful for an improved measurements [22]. Special-designed prostheses [23], surgeon-controlled table, and advanced fluoroscopic imaging techniques [24]. would improve the biomechanical and physiological hip reconstruction and subsequently enhance the radiographic and clinical outcomes.

Interestingly, both the ALA and PLA are comparable in restoring the postoperative offset. Previous literatures evidenced that the offset parameters (FO, $A O$, and $G O)$ have significant impacts on the postoperative function rehabilitation and preoperative templating, and the proper intraoperative verification is helpful to achieve an accurate offset restoration, yielding a better abductor strength as well as a long-term successful prognosis $[25,26]$. Our data showed that postoperative AO, FO and GO was lower in the ALA group than that in the PLA group. These results indicate that both the ALA and the PLA are possibly proper for the unilateral primary THA in selected conditions regarding the offset restoration in line with the general investigations [26].

The DDH represents abnormalities of both acetabulum and femur, requiring special techniques to reconstruct its physiological biomechanical $[27,28]$. Our data with $81 \mathrm{DDH}$ patients (including 49 young females) showed that the DDH patients tend to yield the more postoperative discrepancy and the DDH diagnosis is a significant contributor to the postoperative LLD. Previous literatures demonstrated that the discrepancy and offset might result from an improper position of the femoral stem and acetabular cup, and special thinner, therefore, shorter stems with more non-sprouted sleeves might be more suitable [29, 30]. Arthroplasty surgeons are recommend paying more attention to the perioperative plan in consideration of high risk of postoperative LLD. External obturator footprint, robotic-assisted intervention, gait training, and 3D-printed individual templates provide personal plan to improve the THA outcomes for DDH patients [31].

Moreover, the ALA seems cause the less soft tissue damage than the PLA group for the primary THAs with the comparable safety and efficacy. Previous research showed that the joint degeneration is associated with the body weight of patients $[32,33]$. However, our data revealed that $\mathrm{BMI}$ is not a significant contributor to $\operatorname{LLD}(p=0.126 ; \beta=0.30,95 \% \mathrm{Cl},-0.08 \mathrm{~mm}$ to $0.67 \mathrm{~mm})$ but an increased postoperative discrepancy is noticed in patients with a higher BMI (Figure. 3 ). Therefore, controlling weight might still be helpful for obese patients before the elective surgeries. More studies are necessary 
to identify such a possible beneficial effect of the body weight controlling strategy for the ultimate success of THAs.

Several limitations should be noticed in this study. First, the retrospective nature of the study might not allow us to draw conclusive statements. Second, significant intergroup difference of patient age was observed with divergent sample size, which seems as an unbalanced group (PLA $=200$ vs ALA $=66)$ of patients with what appears to be predominantly DDH $(81 / 266, A L A=22, P L A=59)$. Third, the follow-up is relatively short, more trails with high-level evidence were needed to confirm the findings from the represent study. Strengths of this study include the detailed comparison and analysis of radiographic data performed in a standard manner with a relatively large and homogenous patients operated by the identical highly experienced surgeon team from a single institution.

\section{Conclusions}

In conclusion, our study illustrates that the ALA might be superior to PLA in controlling short-term postoperative LLD among some of the unilateral primary THA. In regard to the FO and GO restoration and medical quality including LOS, both approaches are comparable. More investigations are required to provide convincing favorable evidence to further compare the ALA and PLA in primary THA.

\section{Abbreviations}

Total hip arthroplasty, THA; lower limb discrepancy, LLD; anterolateral approach, ALA; posterolateral approach, PLA; hip osteoarthritis, HOA; developmental dysplasia of the hip, DDH; aseptic vascular necrosis, AVN; length of stay, LOS; femur neck fracture, FNF; DAA; direct lateral approach, DLA; direct superficial approach, DSA; body mass index, BMl; American Society of Anesthesiologists, ASA; diabetes metabolism, DM; coronary heart disease, CHD; femoral offset, FO; acetabular offset, AO; global offset, GO; Picture Archiving and Communication System, PACS; center of rotation, COR; standard deviation, SD; confidence interval, $\mathrm{Cl}$; N, number; S.E., standard error.

\section{Declarations}

\section{Ethics approval and consent to participate}

Ethical review approval was obtained from the Human Subject Committee at Ethics Committee of Peking University Shenzhen Hospital (No.2019038). The research was conducted retrospectively, we have obtained participants' consent and ethics approval from ethics committee.

\section{Consent for publication}

Not applicable.

\section{Availability of data and materials}


Data are available from the authors upon reasonable request and with permission of Department of bone and joint in Peking University Shenzhen Hospital.

\section{Competing interests}

All of the authors have disclosed potential conflicts of interest, which may include receipt of payment, either direct or indirect, institutional support, or association with an entity in the biomedical field which may be perceived to have potential conflict of interest with this work. No conflicts reported in regards to this manuscript.

\section{Funding}

This study was supported by grants from National \& Local Joint Engineering Research Center of Orthopaedic Biomaterials (XMHT20190204007), Shenzhen High-level Hospital Construction Fund, Shenzhen Key Medical Discipline Construction Fund (No. SZXK023), Shenzhen "San-Ming" Project of Medicine (No. SZSM201612092), Research and Development Projects of Shenzhen (No. Z2021N054 and No.JCYJ20190809110807421), National Natural Science Foundation of China (No. 82001319), Guangdong Basic and Applied Basic Research Foundation (No. 2021A1515012586, 2019A1515011290 and 2019A1515110983), Bethune Charitable Foundation and CSPC Osteoporosis Research Foundation Project (No. G-X-2020-1107-21).

\section{Author contributions}

Ao Xiong and Guoqing Li participated in the design and prepared the manuscript, they contribute equally to this work and they were listed as cooperated first authors. Guoqing Li and Su Liu participated in the data collection and radio assessment; Chang Xu participated in execution and analysis of the work presented. Deli Wang and Hui Zeng participated in the design and revision, and reviewed the final manuscript, they contribute equally to this work and they were listed as corresponding authors.

\section{Acknowledgements}

The authors sincerely acknowledge the entire staff of the Department of Bone \& Joint Surgery, Peking University Shenzhen Hospital, who offered their assistance throughout the course of this study.

\section{References}

1. Gao L, Han Z, Xiong A. Total Hip Arthroplasty or Hemiarthroplasty for Hip Fracture. N Engl J Med. 2020;382(11):1072-3.

2. Viamont-Guerra MR, Saffarini M, Laude F. Surgical Technique and Case Series of Total Hip Arthroplasty with the Hueter Anterior Approach for Crowe Type-IV Dysplasia. J Bone Joint Surg Am. 2020;102(21S Suppl 1):99-106. 
3. Kobayashi S, Saito N, Horiuchi H, lorio R, Takaoka K. Poor bone quality or hip structure as risk factors affecting survival of total-hip arthroplasty. Lancet. 2000;355(9214):1499-504.

4. Petersen WP Jr, Teo GM, Friedlander S, Schwarzkopf R, Long WJ. The Implications of Aging Population Demographics on the Delivery of Primary Total Joint Arthroplasty in a Bundled Payment System. J Bone Joint Surg Am. 2020;102(19):1679-86.

5. Mutlu T, Daşar U, Bicimoglu A. Does THA Improve Social Status Among Turkish Women With Developmental Dysplasia of the Hip? Clin Orthop Relat Res. 2018;476(9):1696-703.

6. Warschawski Y, Garceau SP, Joly DA, Kuzyk P, Gross A, Safir O. The Effect of Femoral Head Size, Neck Length, and Offset on Dislocation Rates of Constrained Acetabular Liners. J Arthroplasty, 2020.

7. Angerame MR, Fehring TK, Masonis JL, Mason JB, Odum SM, Springer BD. Early Failure of Primary Total Hip Arthroplasty: Is Surgical Approach a Risk Factor? J Arthroplasty. 2018;33(6):1780-5.

8. Thorne TJ, Nishioka ST, Andrews SN, Mathews KA, Nakasone CK. Comparison of Component Placement Accuracy Using Two Intraoperative Fluoroscopic Grid Technologies During Direct Anterior Total Hip Arthroplasty. J Arthroplasty. 2020;35(12):3601-6.

9. Krueger DR, Guenther KP, DemI MC, Perka C, Mechanical failure of 113 uncemented modular revision femoral components. Bone Joint J, 2020. 102-b(5): p. 573-579.

10. Brun OL, Sund HN, Nordsletten L, Röhrl SM, Mjaaland KE. Component Placement in Direct Lateral vs Minimally Invasive Anterior Approach in Total Hip Arthroplasty: Radiographic Outcomes From a Prospective Randomized Controlled Trial. J Arthroplasty. 2019;34(8):1718-22.

11. Taunton MJ, Trousdale RT, Sierra RJ, Kaufman K, Pagnano MW. John Charnley Award: Randomized Clinical Trial of Direct Anterior and Miniposterior Approach THA: Which Provides Better Functional Recovery? Clin Orthop Relat Res. 2018;476(2):216-29.

12. Wirries N, Ezechieli M, Floerkemeier T, Windhagen $\mathrm{H}$, Skutek $\mathrm{M}$, Proceeding from direct lateral to anterolateral approach in total hip arthroplasty: A closer look on radiological and clinical aspects. J Orthop, 2020. 21: p. 10-13.

13. Innmann MM, Streit MR, Kolb J, Heiland J, Parsch D, Aldinger PR,.. . Merle C. Influence of surgical approach on component positioning in primary total hip arthroplasty. BMC Musculoskelet Disord. 2015;16:180.

14. Chen W, Sun JN, Zhang Y, Zhang Y, Chen XY, Feng S. Direct anterior versus posterolateral approaches for clinical outcomes after total hip arthroplasty: a systematic review and meta-analysis. J Orthop Surg Res. 2020;15(1):231.

15. Stangl-Correa P, Stangl-Herrera W, Correa-Valderrama A, Ron-Translateur T, Cantor EJ, PalacioVillegas JC. Postoperative Failure Frequency of Short External Rotator and Posterior Capsule With Successful Reinsertion After Primary Total Hip Arthroplasty: An Ultrasound Assessment. J Arthroplasty. 2020;35(12):3607-12.

16. Frisch NB, Courtney PM, Darrith B, Copeland LA, Gerlinger TL. Veterans Undergoing Total Hip and Knee Arthroplasty: 30-day Outcomes as Compared to the General Population. J Am Acad Orthop Surg, 2020. 
17. Park CW, Lim SJ, Cha YT, Park YS. Total Hip Arthroplasty With Subtrochanteric Shortening Osteotomy in Patients With High Hip Dislocation Secondary to Childhood Septic Arthritis: A Matched Comparative Study With Crowe IV Developmental Dysplasia. J Arthroplasty. 2020;35(1):204-11.

18. Ihaka R, Gentleman R. R: A Language for Data Analysis and Graphics. Journal of Computational Graphical Statistics. 1996;5(3):299-314.

19. Pocock SJ, Stone GW. The Primary Outcome Is Positive - Is That Good Enough? N Engl J Med. 2016;375(10):971-9.

20. Mavčič B, Dolinar D, Pompe B, Antolič V. Patient-dependent risk factors for self-perceived leg length discrepancy after total hip arthroplasty. Eur J Orthop Surg Traumatol. 2019;29(4):793-9.

21. Kishimoto Y, Suda H, Kishi T, Takahashi T. A low-volume surgeon is an independent risk factor for leg length discrepancy after primary total hip arthroplasty: a case-control study. Int Orthop. 2020;44(3):445-51.

22. Grosso P, Snider M, Muir JM. A Smart Tool for Intraoperative Leg Length Targeting in Total Hip Arthroplasty: A Retrospective Cohort Study. Open Orthop J. 2016;10:490-9.

23. Gabor JA, Singh V, Padilla JA, Schwarzkopf R, Davidovitch RI. Outcomes with Two Tapered Wedge Femoral Stems in Total Hip Arthroplasty Using an Anterior Approach. J Orthop. 2020;22:341-5.

24. Delagrammaticas DE, Ochenjele G, Rosenthal BD, Assenmacher B, Manning DW, Stover MD. Intraoperative evaluation of acetabular cup position during anterior approach total hip arthroplasty: are we accurately interpreting? Hip Int. 2020;30(1):40-7.

25. Kiernan S, Geijer M, Sundberg M, Flivik G. Effect of symmetrical restoration for the migration of uncemented total hip arthroplasty: a randomized RSA study with 75 patients and 5-year follow-up. J Orthop Surg Res. 2020;15(1):225.

26. Enke O, Levy YD, Bruce WJ. Accuracy of leg length and femoral offset restoration after total hip arthroplasty with the utilisation of an intraoperative calibration gauge. Hip Int. 2020;30(3):296-302.

27. Tang L, Chen M, Li G, Luo Z, Ji X, Zhang X,... Shang X, [Effectiveness of proximal femur reconstruction combined with total hip arthroplasty for Crowe type âष£ developmental dysplasia of hip]. Zhongguo Xiu Fu Chong Jian Wai Ke Za Zhi, 2020. 34(6): p. 683-688.

28. Ohsawa S, Koide S, Shibuya T. Mid-term results of total hip arthroplasty for relapsed osteoarthritis after joint-preserving operations for coxarthritis compared to primary total hip arthroplasty. Eur J Orthop Surg Traumatol, 2020.

29. Al-Amiry B, Mahmood S, Krupic F, Sayed-Noor A. Leg lengthening and femoral-offset reduction after total hip arthroplasty: where is the problem - stem or cup positioning? Acta Radiol. 2017;58(9):112531.

30. Ueoka K, Kabata T, Kajino Y, Inoue D, Ohmori T, Ueno T,.. . Tsuchiya H. Patient-reported outcomes following primary total hip arthroplasty in Crowe type III or IV developmental dysplasia are comparable to those in Crowe type I: a case-control study of 96 hips with intermediate-term followup. BMC Musculoskelet Disord. 2020;21(1):344. 
31. Perets I, Walsh JP, Mu BH, Mansor Y, Rosinsky PJ, Maldonado DR,.. . Domb BG, Short-term Clinical Outcomes of Robotic-Arm Assisted Total Hip Arthroplasty: A Pair-Matched Controlled Study. Orthopedics, 2020: p. 1-7.

32. Okuzu Y, Goto K, Shimizu Y, Kawai T, Kuroda Y, Matsuda S. Sacroiliac joint degeneration is common in patients with end-stage hip osteoarthritis secondary to unilateral developmental dysplasia of the hip: Factors associated with its severity and laterality. J Orthop Sci, 2020.

33. Al-Amiry B, Pantelakis G, Mahmood S, Kadum B, Brismar TB, Sayed-Noor AS. Does body mass index affect restoration of femoral offset, leg length and cup positioning after total hip arthroplasty? A prospective cohort study. BMC Musculoskelet Disord. 2019;20(1):422.

\section{Figures}

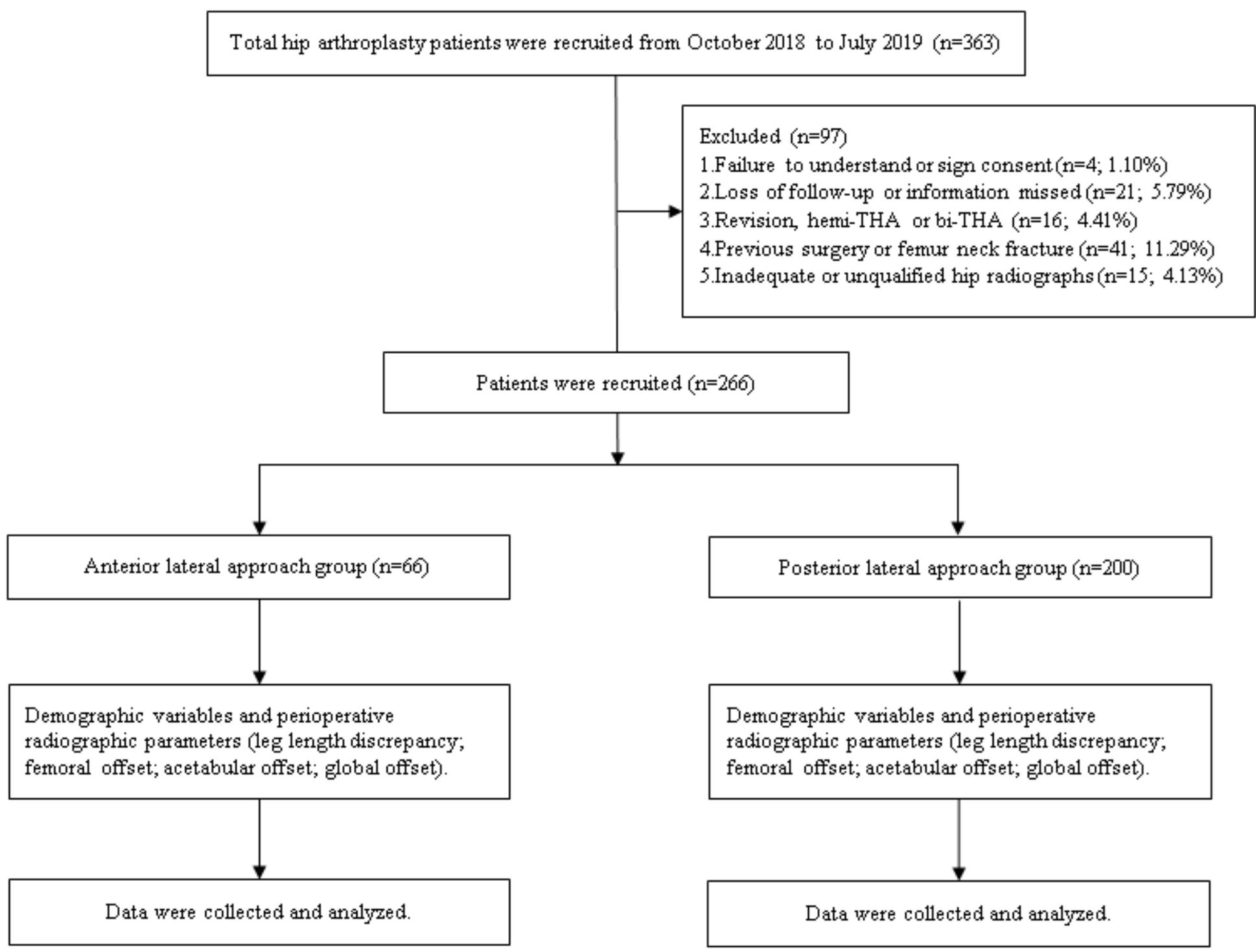

\section{Figure 1}

Flowchart illustrating participation selection 

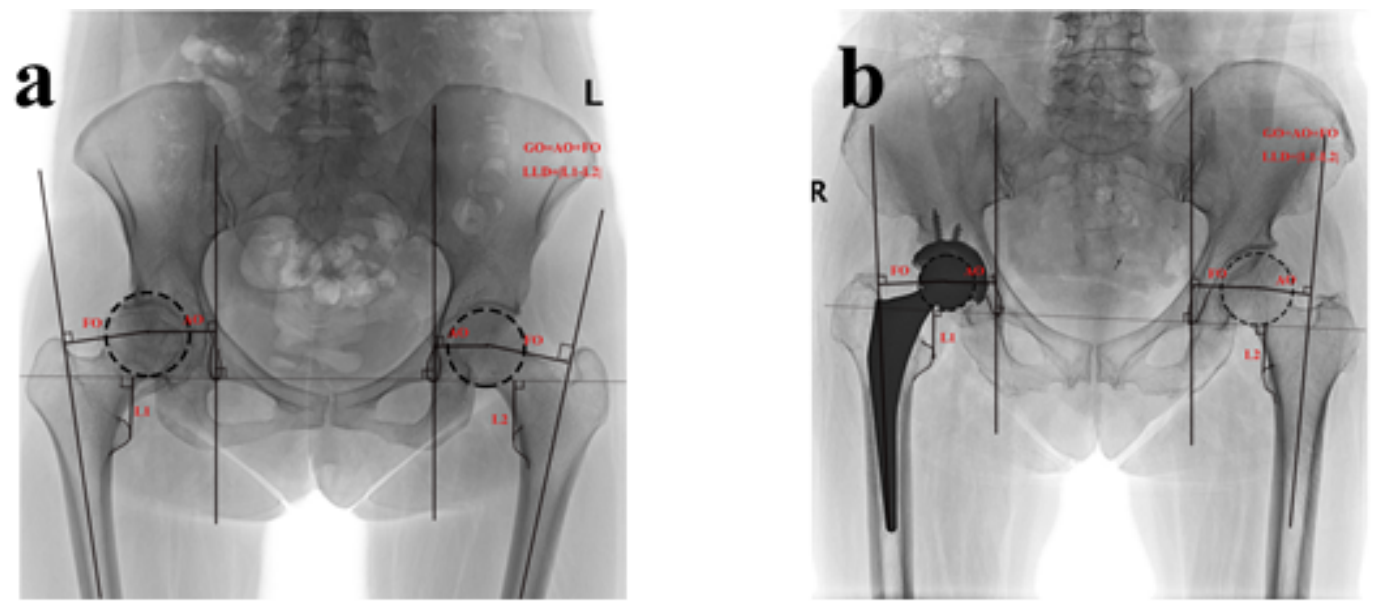

\section{Figure 2}

Illustrative preoperative (a) and postoperative (b) anteroposterior pelvic radiograph

a

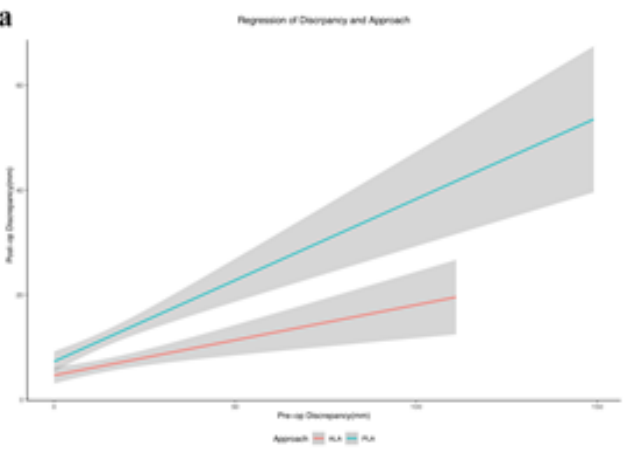



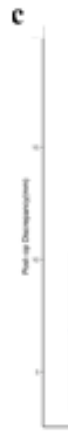

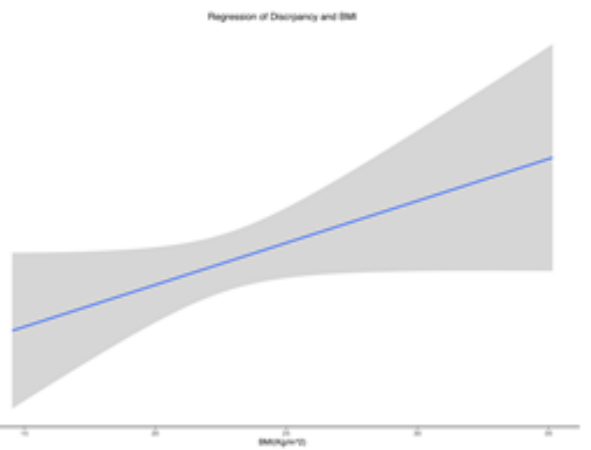

Figure 3

Regression curves of postoperative discrepancy and preoperative LLD (a), diagnosis (b) and BMI (c)
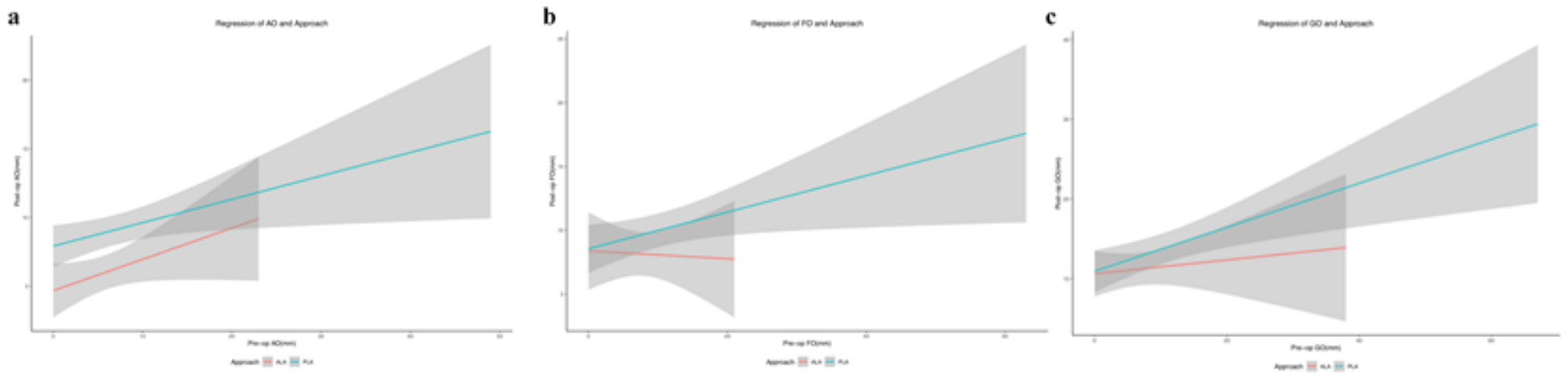

Figure 4

Regression curves of preoperative offset and postoperative offset (AO (a), FO (b), and GO (c)) 